INVESTIGACIÓN DIDÁCTICA

\title{
Implantación de la nueva asignatura "Robótica" en Enseñanza Secundaria y Bachillerato
}

\author{
Gabriel Ocaña Rebollo, Isabel Ma Romero Albaladejo, Universidad de Almería” \\ Francisco Gil Cuadra y Antonio Codina Sánchez
}

RESUMEN: En el presente artículo se presenta el material curricular y la metodología de trabajo desarrollados para la implantación de las asignaturas de "Robótica", de nueva creación para la Enseñanza Secundaria. Se describe el paradigma de Educación STEM y el Método de Proyectos como enfoque metodológico que sustenta la propuesta. Se muestra el potencial de las asignaturas y de los materiales desarrollados para fomentar la motivación y el aprendizaje de los estudiantes

Palabras clave: Robótica Educativa; Educación STEM; Método Proyectos; Materiales Curriculares.

$* * * *$

\section{ABSTRACT: Implementing new subject "Robotics" in Secondary Education}

In the present article, curricular materials and methodology developed for the implementation of "Robotics" subjects, newly created for Secondary Education, are presented. We describe the paradigm of STEM Education and the Project Method as a methodological approach that supports the proposal. The potential of these subjects, together with the materials developed, to foster motivation and learning in students is exposed.

Keywords: Educational Robotics; STEM Education; Project Method; Curricular Material.

RÉSUMÉ: Mise en œuvre des nouveaux matières "Robotique" dans l'Enseignement Secondaire

Cet article présente le matériel pédagogique et la méthodologie développé pour l'implantation des matières de «robotique», nouvellement crées pour l'enseignement secondaire. Nous décrivons le paradigme de l'éducation STEM et la Méthode du Projet comme une approche méthodologique qui prend en charge le matériel pédagogique et la méthodologie qui sous-tend la proposition. II montre le potentiel des matières et des matériaux développés pour favoriser la motivation et l'apprentissage des élèves.

Mots clé: Robotique pour l'éducation, STEM, Méthode du Projet, Matériel Pédagogique

\footnotetext{
* Gabriel Ocaña Rebollo. IES Turaniana. Avenida de los estudiantes, 86. Roquetas de Mar, Almería. 04740. gabriel.ocana@ hotmail.es; Isabel Mª Romero Albaladejo. Universidad de Almería. Edificio Central. La Cañada de San Urbano s/n, 04120. Almería. imromero@ual.es; Francisco Gil Cuadra. Universidad de Almería. Edifico A. La Cañada de San Urbano s/n, 04120. Almería. fgil@ual.es; Antonio Codina Sánchez. Universidad de Almería. Edificio A. La Cañada de San Urbano s/n, 04120. Almería. acodina@ual.es

$\bowtie$ Artículo recibido el 25 de Enero de 2013 y aceptado el 6 de Abril de 2015.
} 


\section{Introducción}

A lo largo del último siglo, la Tecnología ha ido adquiriendo una importancia progresiva en el funcionamiento de la sociedad y en la vida de las personas. Según la normativa vigente, las asignaturas de Tecnología en Educación Secun daria tienen como una de sus finalidades acercar al alumnado a esta realidad (Ministerio de Educación, 2007a).

Dentro de estas asignaturas, los Proyectos de Tecnología normalmente representan la parte que resulta más atractiva al alumnado. El objetivo de cada Proyecto es construir un objeto técnico para poner en práctica los conocimientos propios de la materia siguiendo el método Proyecto-Construcción. Sin embargo, al desarrollar el Proyecto de forma tradicional nos encontramos que se suelen abarcar áreas de conocimiento limitadas, debido a condicionamientos de tiempo y herramien tas disponibles, en detrimento del sentido de experimentación y de adquisición de nuevo conocimientos. Esto sucede especialmente en los últimos cursos de Enseñanza Secundaria Obligatoria (ESO) y en Bachillerato. Y los jó venes de nuestros días, que viven rodeados de aparatos altamente tecnológicos, difícilmente se sienten atraídos por este tipo de montajes sencillos.

Como consecuencia de estas limitacione prácticas, surgió la iniciativa de utilizar la Robótica Educativa y adaptarla a la Enseñanza Secundaria como una asignatura oficial del currículo.

Las áreas de conocimiento involucradas en Robótica (Gura, 2011) forman parte del desarrollo curricular establecido para la enseñanz de Tecnología en la ESO y Bachillerato (Ministerio de Educación, 2007a, 2007b):

- Mecánica: Construyendo la estructura de un robot los estudiantes aprenden concepto como estabilidad estructural, y transmisión y transformación de movimientos.

Electrónica: Interconectando los sensores y actuadores se ponen en práctica circuito electrónicos, manejo de motores y conceptos de retroalimentación y control.
- Informática: Programando el comportamiento del robot los estudiantes analizan las tareas necesarias para que pueda decidir las acciones a realizar a partir de información externa.

Por lo tanto, la robótica educativa permite desarrollar los objetivos, contenidos y competencias propios de las asignaturas de Tecnología (Ocaña, 2012). Además, la construcción y programación de robots permite a los estudiantes experimentar y comprender conocimientos interdisciplinares de Matemáticas, Física, Informática, etc., como se expondrá más adelante.

Otra característica que tiene la Robótica Educativa es favorecer el proceso de enseñanza-aprendizaje integrando el aspecto teórico con el práctico, manteniendo la atención del estudiante y desarrollando un pensamiento sistémico (Ruiz-Velasco, 2007). El hecho de que los robots se perciban como algo asociado a la alta tecnología, e incluso a la ciencia ficción, nos permite incentivar los estudios científico-tecnológicos mediante esta materia.

Por todas estas razones se propuso la creación e implantación de las nuevas asignaturas de Robótica, inexistentes hasta la fecha en Andalucía. Tras su aprobación, se impartieron por primera vez en los cursos 2008/09 ( $4^{\circ} \mathrm{de}$ la ESO) y $2010 / 11$ ( $2^{\circ}$ de Bachillerato) en el IES Turaniana de Roquetas de Mar, Almería. Posteriormente se han ido utilizando los materiales y metodología desarrollados en varios Institutos de la provincia de Almería, bien como asignatura con entidad propia o dentro del marco de otras asignaturas (normalmente Tecnología)

En este trabajo se presentan las asignaturas de Robótica de nueva creación. Se expone el marco teórico que las fundamenta y se explica cómo ha sido su implantación, atendiendo a los materiales curriculares que se han desarrollado, los cursos donde se imparten, la metodología de enseñanza-aprendizaje y las actividades que se realizan. Finalmente, se exponen algunos de los resultados obtenidos y las líneas de trabajo futuras.

\section{Marco Teórico}

El marco teórico que fundamenta las asignaturas de Robótica es la aplicación del paradigma de Educación STEM en combinación con el Método de Proyecto-Construcción. Esto implica un aprendizaje orientado a la resolución de problemas, a través del desarrollo de un Proyecto con los métodos propios de Ingeniería para generar un producto tecnológico tangible (en nuestro caso un robot), integrando conocimientos multidisciplinares de Ciencia y Tecnología.

\section{Educación STEM}

El término "STEM" es el acrónimo de los términos en inglés Science, Technology, Engi neering and Mathematics. El concepto "Educación STEM" se ha desarrollado como una nueva manera de enseñar conjuntamente Ciencias y Tecnología con dos características diferenciadoras (Sanders, 2009):

- Enseñanza-aprendizaje de Ciencia, Tec nología, Ingeniería y Matemáticas de maner conjunta e integrada, en lugar de como áreas de conocimiento compartimentadas.

- Con un enfoque de Ingeniería en cuanto al desarrollo de conocimientos dirigidos siempre la resolución de problemas tecnológicos reales.

La relación existente entre las Matemáticas, Ciencia y Tecnología es inherente a esta disciplinas. De lo que se trata es de provocar intencionadamente situaciones que permitan aprender de manera simultánea e integrada conceptos de estas materias. Esto se lleva a cabo en un proceso práctico de diseño y resolución de problemas, tal y como se hace en Ingeniería en el mundo real (Fortus et al., 2005).

Por otro lado, la esencia de la Ingeniería es diseñar y llevar a la práctica esa solución en la construcción de un objeto técnico. Éste es un proceso complejo que consiste en aplicar conocimientos científico-tecnológicos diversos par solucionar un problema real (Schön, 1987).

La evolución educativa que supone la Educación STEM en el s. XXI es que la Ingeniería y sus métodos se abren paso en el currículo de la Educación Secundaria, de igual modo que la Ciencia y los procesos asociados al Método Científico (fundamentalmente el método hipotético-deductivo) se han incorporado al currículo a lo largo del siglo XX, (Capraro y Slough, 2009).

La Educación STEM está basada en principios del constructivismo (Bruning et al., 2004) Por tanto, la pedagogía de la Educación STEM está centrada en el estudiante y en el conocimiento. Además, cuando se utiliza en grupos de aprendizaje se proporciona un entorno de interacción social (Bransford et al., 2000).

En nuestro caso, aplicamos una extensión del constructivismo conocido como "Construccionismo" (Papert, 1980), que añade el hecho de que construir nuevo conocimiento es más efectivo cuando los estudiantes están involucrados en la construcción de objetos que le son significativos. Se resalta por tanto la importancia del aspecto constructivo manual para estimular el pensamiento creativo y favorecer la construcción formal de nuevos conocimientos.

Hay cada vez más evidencias de que los procedimientos STEM mejoran los aprendizajes, haciéndolos más efectivos y funcionales (Hartzler, 2000; Fisher et al., 2005; Wai et al., 2010). Se pueden resumir los beneficios de un sistema de Educación STEM en (Laboy-Rush, 2011; Mastascusa, et al., 2011):

Transferencia de los conocimientos y habilidades a problemas del mundo real.

- Incremento de la motivación para aprender.

- Retención a largo plazo, una vez aprendido el conocimiento.

- Mejora los aprendizajes posteriores de conocimientos relacionados.

Es de destacar que el aprendizaje se mejora en términos generales y para una mayoría de alumnos, no solo con los más cualificados y/o futuros científicos e ingenieros (Drew, 2011).

No obstante, también hay retos para la implementación con éxito de una Educación STEM. Algunos son comunes a cualquier proceso de reforma educativa, como el tiempo de preparación adicional para los docentes, la necesidad de materiales y recursos específicos, o 
las actitudes del profesorado hacia los cambios en su práctica docente (Harland, 2011; Capraro et al., 2010)

Otros desafíos más específicos de este tipo de educación son, según Satchwell y Loepp (2002):

- La integración del currículo de Matemáticas, Ciencias y Tecnología es una tarea difíci que requiere el desarrollo de un marco curricular específico.

- Se necesitan unas instalaciones adecuadas.

- Es preciso proporcionar una formación STEM para los Profesores de Matemáticas, Ciencias y Tecnología.

\section{Aprendizaje Basado en Proyectos. \\ Método Proyecto-Construcción}

Aunque los estudiantes aprenden mejor cuando se les anima a construir su propio conocimiento sobre el mundo que les rodea, con frecuencia la educación en Ciencias se bas en el aprendizaje abstracto de fórmulas y leyes (Satchwell y Loepp, 2002). Generalmente no se hacen conexiones con el mundo real y el aprendizaje es independiente de las experiencias cotidianas. Necesitan aprender a toma decisiones y a resolver problemas, en lugar de simplemente almacenar colecciones de dato (Kolodner et al., 2003). La visión del Método d Proyectos o Aprendizaje Basado en Proyectos es darle la vuelta a esta situación, involucrando a los estudiantes en proyectos del mundo real en los que aprendan las fórmulas y leye que explican cómo funciona el mundo que nos rodea (Hernández y Ventura, 1992; Thomas, 2000; Capraro y Slough, 2009; Criado y García, 2011). Tiene raíces en el constructivismo, construccionismo y aprendizaje cooperativo/ colaborativo (Grant, 2002)

Existen distintos tipos de proyectos (Thomas, 2000): Información, Acción y Producción. En las asignaturas de Tecnología se aplica el Método de Proyecto-Construcción (Real Decreto 1631/2006), que pertenece a la tipología de Proyecto Producción. Está orientado a la construcción de prototipos técnicos que permitan desarrollar el conocimiento propio de la asignatura de Tecnología como elemento integrador de conocimientos científico-técnicos. Tiene por tanto un carácter interdisciplinar (Pozuelos y Rodríguez, 2008; Lacueva, 2008).

El hecho de que el Aprendizaje Basado en Proyecto-Construcción sea el utilizado en las asignaturas de Tecnología, junto con los beneficios que se consiguen combinando este tipo de aprendizaje con la Educación STEM (Fortus y otros, 2005), ha determinado que en el diseño de las nuevas asignaturas de Robótica se haya optado por integrar los dos paradigmas: la novedosa Educación STEM y el conocido Método Proyecto-Construcción

\section{Robótica como Asignatura en \\ Enseñanza Secundaria}

La Robótica como disciplina de estudio es algo complejo. Habitualmente se cursa en asignaturas de últimos años de Ingeniería y, por consiguiente, la mayoría del material de enseñanza disponible (robots y contenidos) está enfocado a la Universidad: montaje basado en tuercas y soldadura, programación en lenguajes sofisticados, así como un coste desorbitado para un centro de Secundaria.

A la hora de diseñar las asignaturas de Robótica en Secundaria se tuvieron que superar dos obstáculos. Uno fue encontrar recursos adecuados a este nivel y preparar actividades de enseñanza-aprendizaje basadas en esos recursos. Otro fue implantar el novedoso enfoque de Educación STEM

El material curricular para las asignaturas de Robótica se ha creado a medida con un considerable esfuerzo de diseño: programaciones, unidades didácticas, actividades de aula, material para el profesor, etc. Todo este material curricular ha sido publicado por la Consejería de Educación de la Junta de Andalucía (Ocaña, 2012), y enviado gratuitamente a centros públicos y concertados de Andalucía, así como a diversos organismos e instituciones públicas. Parte de estos materiales curriculares se han realizado dentro del marco de un Proyecto de Elaboración de Materiales Curriculares y Re- cursos Didácticos sobre Robótica Educativa ${ }^{1}$ coordinado por el primer autor de este artículo y financiado por la misma Consejería.

En lo que a Educación STEM se refiere, es una novedad en nuestro sistema educativo crea una asignatura con este enfoque interdisciplina cuando el currículo oficial establece claramente asignaturas independientes de Matemáticas, Fsica, Tecnología o Informática. Nuestra solución se aprovecha de que la Robótica es una materia interdisciplinar por naturaleza. Sería imposible estudiar por separado los componentes matemá ticos, físicos, tecnológicos, o informáticos en el diseño, construcción y programación de robots.

La coexistencia de la Robótica como asignatura STEM con el resto de asignaturas se plantea como un complemento que nunca sustituye ni entra en conflicto con las asignatura oficiales. En algunas ocasiones, los estudiantes aprenderán contenidos nuevos que según el currículo oficial estudiarán posteriormente. Es e caso de los tipos de movimiento relativo y absoluto en Física, propio de primeros cursos de carreras universitarias. Este tipo de movimiento lo manejan cuando un robot en movimiento lanza un objeto del que hay que controlar la velocidad y/o trayectoria.

En otras ocasiones, los estudiantes aprenderán a poner en práctica en contextos reales aquellos conceptos teóricos que solo sabían aplica para resolver ejercicios de clase. Un ejemplo anecdótico puede ser la exclamación de un alumno de $1^{\circ}$ de Bachillerato intentando averiguar por que un robot se tomaba de modo diferente la mism curva según fuesen las condiciones del suelo:

"Ahora entiendo de verdad lo que significa fuerza de rozamiento"

Lo curioso es que un alumno en $1^{\circ}$ de $\mathrm{Ba}-$ chillerato de Ciencias lleva ya dos años estudiando y resolviendo problemas sobre la fuerza de rozamiento en Física.

\section{Cursos de las Asignaturas de Robótica}

La normativa vigente en Andalucía permite que los Institutos de Educación Secundaria puedan ofertar asignaturas de libre configuración en diferentes cursos de la ESO y Bachillerato (Ministerio de Educación, 2007a, 2007b; Consejería de Educación, 2007, 2008).

Así, se diseñaron las asignaturas de Robótica como asignaturas optativas de libre configuración. Por tanto, como asignaturas independientes con entidad propia, anuales, evaluables y que forman parte del currículo con sus propios objetivos y contenidos educativos. En concreto, se implantó la asignatura "Robótica" como Optativa de Libre Configuración en $4^{\circ} \mathrm{de}$ la ESO (2008/09) y "Robótica Avanzada" en $2^{\circ}$ de Bachillerato (2010/11) en el IES Turaniana de Roquetas de Mar, Almería.

La razón de elegir $4^{\circ}$ de la ESO y Bachillerato es que los alumnos se agrupan en modalidades según las asignaturas optativas que cursan. Por lo tanto, los alumnos que eligen Robótica ya tienen una predisposición positiva hacia el mundo de la Ciencia y la Tecnología. Esto facilita su motivación con un recurso educativo novedoso y con un gran componente tecnológico $^{2}$. Por otra parte, al estar en cursos terminales tienen un conocimiento global de Tecnología, Matemáticas y Física, lo que facilita que la Robótica ayude a los alumnos de Ciencias a desarrollar mejor sus conocimientos de manera integrada y significativa.

\section{Factores Contextuales del Proceso}

\section{de Enseñanza-Aprendizaje}

A continuación se expone una serie de factores que creemos que influyen significativamente en el desarrollo de las asignaturas de Robótica y en los resultados obtenidos.

Proyecto MTT-003/10 de la Consejería de Educación de la Junta de Andalucía: "Robótica Educativa en centros TIC. Una plataforma educativa y actividades de enseñanza-aprendizaje con software libre".

2 Lo curioso es que a pesar del enfoque inicial, desde su implantación siempre hay en torno a un 30\% de alumnos de robótica que no son de Ciencias. 


\section{Papel del Profesor}

Una pieza organizativa fundamental es e equipo de trabajo que forman los estudiantes. Todas las tareas se realizan en grupos de dos ó tres personas. A cada equipo se le asigna un robot en exclusividad durante todo el curs escolar del que son responsables, y tienen que colaborar en la organización de los cientos de piezas que tienen a su disposición.

La toma de decisiones en cada equipo es por consenso, teniendo que ser responsables de las decisiones que toman ya que la opinión del equipo prevalece.

\section{Resultados Tangibles a Corto Plazo}

Tanto la selección del material de trabajo como el diseño de las actividades se han realizado con la intención de conseguir muchos re sultados a corto plazo. Este enfoque se opone otros posibles como podría ser dedicar grande períodos de tiempo a hacer pocos robots pero muy sofisticados.

Cada actividad tiene una duración variable pero una vez construido el robot básico se programan entre uno y tres comportamientos diferentes por clase. Y los robots más complejos se construyen y programan en 3-4 clases. Solo en las etapa finales de Robótica Avanzada se contemplan actividades cuya duración sea más de 10 clases.

\section{Optatividad de las Asignaturas}

El carácter optativo de las asignaturas hace que normalmente haya de 10 a 20 estudiantes por clase. Estos dos factores, voluntariedad de alumnado en cursar la asignatura y baja ratio alumnado-profesor, consideramos que contribuyen muy positivamente al desarrollo de las clases y al aprovechamiento de las actividade de enseñanza-aprendizaje.
La función del Profesor no es impartir clases magistrales ni dar instrucciones explícitas de cómo se resuelven las tareas. Su papel es más bien de guía sobre unas actividades que ha diseñado previamente de tal modo que, con una presentación adecuada y con la base de conocimientos adquiridos en actividades previas, se consigan desarrollar los aprendizajes previstos a pesar de que los estudiantes dispongan de un grado de libertad muy amplio.

Los equipos elijen su propio camino de resolución de problemas, y el Profesor está atento a los posibles desvíos para reorientarlos. En caso de estar muy perdidos o dedicar demasiado tiempo a una tarea, la reorientación se vuelve cada vez más guiada y directiva.

\section{Atención a la Diversidad}

En el diseño de las asignaturas de Robótica se ha tenido en cuenta la atención a la diversidad en cuanto a variedad de intereses, nivel académico y ritmos de aprendizaje. En el Instituto de Secundaria donde se han implantado, la variedad intercultural es muy grande tanto desde el punto de vista de nacionalidades como de géneros y religiones ${ }^{3}$

Las actividades planteadas no tienen una duración rígida. Es más importante que el aprendizaje sea sólido a que sea rápido y los equipos hacen las actividades según su propio ritmo. de formación en Robótica se han definido diversos "itinerarios" de ampliación de conocimientos que los equipos de estudiantes recorren y profundizan libremente dentro de un mismo curso o incluso entre diferentes cursos: diferentes sistemas de traccion (diferencial, rover, cadenas, artrópodos, bípedos, ...), robots ma-
Además, una vez finalizada la parte básica

\footnotetext{
3 Un ejemplo ilustrativo puede ser la composición del equipo de robótica que participó en el Campeonato del Mundo de Mannheim: una veintena de estudiantes con un $40 \%$ de alumnas, 8 nacionalidades y que profesaban 4 religiones diferentes. La foto del equipo fue expuesta en carteles publicitarios por la provincia de Almería durante el verano de 2012: https://www.facebook. com/photo. php?fbid=161372787329055\&l=4daa9f2ea9
}

nipuladores, dificultades graduadas en cuanto a construcción mecánica y/o programación, diseños mecánicos libres o guiados, programació en lenguajes profesionales como Java, preparación de competiciones de robótica, etc.

\section{FIRST $^{\circledR}$ LEGO $^{\circledR}$ League}

La preparación de la competición de robótica FIRST $^{\circledast}$ LEGO $^{\circledR}$ League (FLL) se ha integrado en clase como una opción más para aquellos alumnos que así lo deseen (Ocaña, 2013). Este tipo de competiciones ofrecen motivació extrínseca adicional para los estudiantes y aumenta sus habilidades de trabajo en equipo (Pisciotta et al., 2010).

Desde el año 2006 se celebra en España la competición internacional FIRST ${ }^{\oplus} L E G O^{\circ}$ League (FLL), realizándose también en An dalucía desde el año 2011. Se trata del mayor programa de Robótica para escolares del mundo, estando presente en más de 70 países Esta competición se creó como un programa de Educación STEM para promover vocaciones científicas y tecnológicas mediante la innovación, la creatividad y el trabajo en equipo (Kelly y Daudelin, 2008).

La participación en FLL abre un abanico mayor de posibilidades a los estudiantes, permitiendo una mayor integración de alum nos con intereses y capacidades muy diversas construcción mecánica, programación, planificación de estrategia, proyecto científico, etc.

En caso de conseguir la clasificación para la fase mundial, los estudiantes deben inclus defender sus proyectos en ingles con presentaciones y turnos de preguntas ante un jurado internacional.

\section{Metodología de Aprendizaje}

En primer lugar, el material de trabajo seeccionado para desarrollar las actividades en las asignaturas de Robótica de ESO y Bachillerato ha sido el robot LEGO Mindstorms.

Estos robots son fáciles de ensamblar y disponen de gran variedad de piezas basadas en los montajes LEGO Technic. Actualmente es el robot educativo más utilizado a nivel mundial, lo que hace que haya gran cantidad de recursos didácticos (en inglés), concursos de Robótica a nivel mundial como la First LEGO League sensores avanzados de fabricantes como HiTechnic $^{6}$ o Mindsensors ${ }^{7}$, e incluso software de diseño asistido por ordenador (LEGO Digital Designer $^{8}$ ).

Por ende, la división LEGO Education enfoca este robot y su manejo con la filosofía de Educación STEM ${ }^{9}$ junto con la metodología pedagógica del construccionismo (Cortés, Arbeláez y Mendoza, 2009; Alimisis y Kynigos, 2009).

Por otro lado, en cuanto a la secuencia de actividades, el pilar fundamental de todo el material curricular desarrollado es que todas y cada una de las unidades de conocimiento que los alumnos deben aprender se trabajan como un proyecto específico de construcción-programación de un robot (Ocaña, 2012), dentro del marco de Educación STEM.

\section{Actividades Iniciales}

Las primeras actividades para asimilar los fundamentos basicos de los robots son de tipo guiado, con prácticas sencillas de dificultad graduada. Esto se hace para introducir progre-

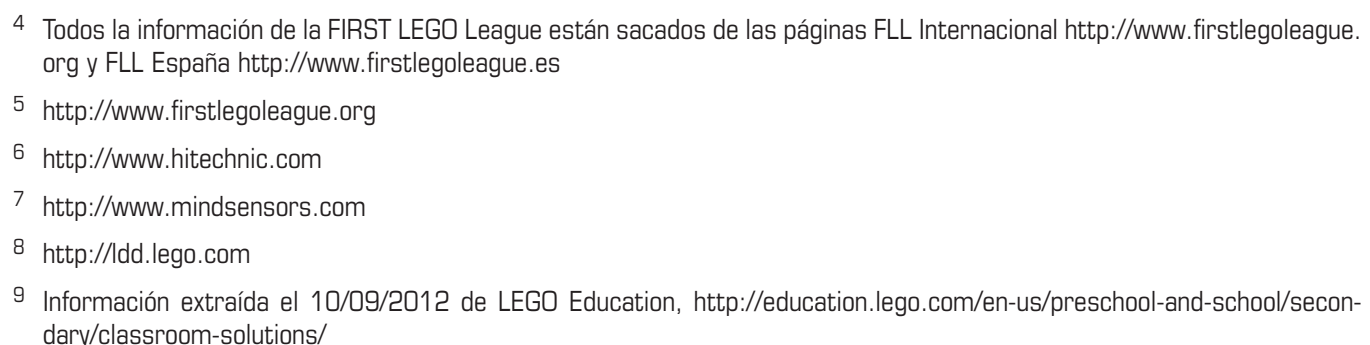
dary/classroom-solutions/ 
sivamente la gran cantidad de conceptos nuevo sobre programación, estructuras, transmisión de movimientos, manejo de motores y sensores, etc. Para garantizar el aprendizaje, cada vez que se termina un bloque de contenidos y antes de pasar al siguiente los alumnos deben realizar robots que engloban todos los conocimientos adquiridos mediante prácticas no guiadas.

Un ejemplo de cómo son estas primera actividades es el control del movimiento de un robot. Primero aprenden de manera guiada a manejar los motores para desplazar el robo adelante y atrás, calcular distancia recorrida, realizar los distintos tipos de curvas, y enlazar movimientos para realizar trayectorias sencillas como cuadrados o circunferencias. Llegados a este punto, se les plantean las siguientes actividades no guiadas:

- Realizar un cuadrado con un tamaño determinado, para que combinen cálculo de distancias con control de trayectorias.

- Hacer una trayectoria con forma de ocho, que es una combinación de rectas y giros.

- Rodear una mesa y acabar en el mismo sitio de partida, para que combinen libremente trayectorias con el suficiente control y cálculo de distancias.

Con esta técnica aprenden primero a controlar el movimiento del robot, y después a manejar los sensores de uno en uno: sonido, luz distancia y contacto.

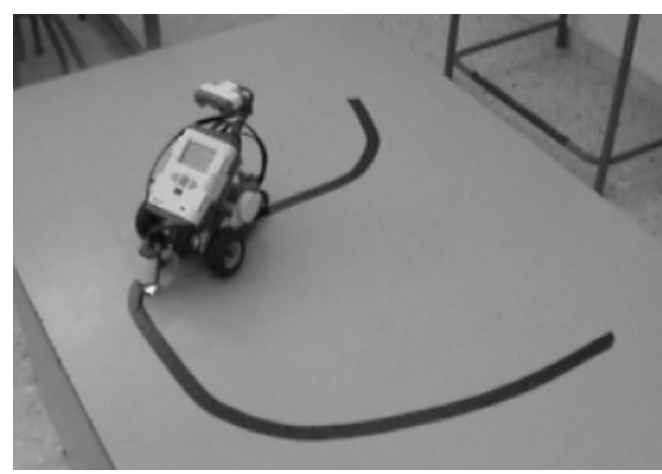

Figura 1. Manejo de sensor de luz: robot seguidor de línea.

Finalmente, cuando han adquirido estos conocimientos básicos, integran todo lo aprendido haciendo robots que combinan varios sensores hasta llegar a robots que utilizan simultáneamente cuatro sensores y tres motores.

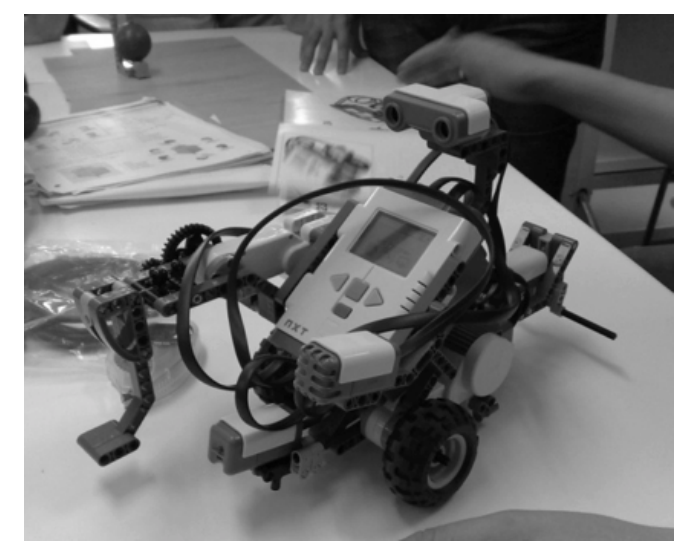

Figura 2. Jugador de Golf: localiza la pelota de su color y la golpea. Utiliza los tres motores y sensores de ultrasonidos, luz y contacto.

\section{Proyectos de Robótica}

Una vez terminada esta fase de iniciación, ya se abordan montajes más sofisticados aplicando de lleno nuestra metodología: Educación STEM y trabajo por Proyectos. Estas actividades constituyen el grueso de las asignaturas de Robótica.

Cada actividad consiste en definir una situación a resolver para que los equipos de trabajo decidan libremente como hacerlo ${ }^{10}$. Ejemplos de estos primeros desafíos que los alumnos resuelven son el Robot Minero y el Perro Guardián (Ver figuras 3 y 4):

El Robot Minero con tracción en las cuatro ruedas debe recorrer una galería de túneles. Cada vez que llega al final de una galería gira la cabeza para elegir el túnel más corto y continuar avanzando hasta el lugar de trabajo.

El Perro Guardián patrulla por un pasillo por el que hay varias puertas. Cada vez que

10 Algunos ejemplos de los robots de aprendizaje que los alumnos realizan se pueden visualizar en la siguiente lista de reproducción: http://www.youtube.com/watch?v=05Jz4pR7Bks\&list=PLFAECFB2277DD13EF\&feature=plpp_play_all

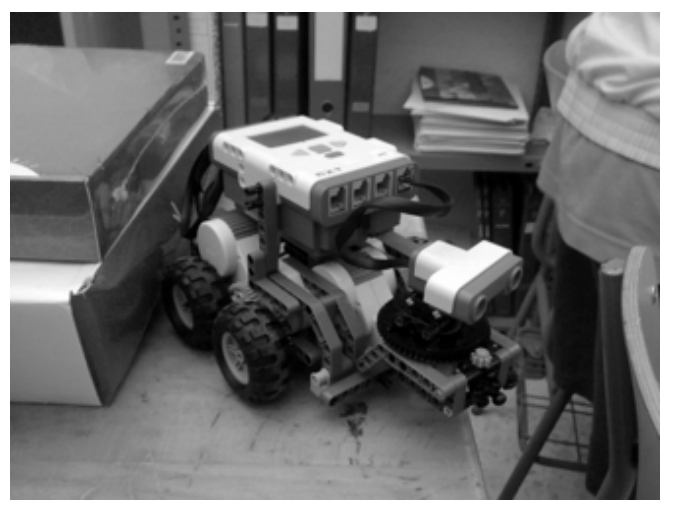

Figura 3. Robot Minero. Debe recorrer un laberinto de túneles y encontrar el final.

pasa por delante de una debe girarse y comprobar si está abierta o cerrada. Si alguna esta cerrada, empieza a ladrar hasta que su dueño le toca el hocico.

\section{Un Ejemplo Concreto de Actividad de Aula}

Para comprender mejor el enfoque seguido en el planteamiento de actividades de aula, se detalla algo más uno de los proyectos que componen la asignatura de Robótica: el proyecto Robot Minero.

El primer paso siempre es la descripción del Profesor sobre la situación que hay que resolve (acompañado de fotos y vídeos) y desglosarlo en problemas de dificultad graduada. En este caso, el primer problema es conseguir que el robot avance por un túnel recto de longitud indefinida, detenerse al final del túnel y gira la cabeza $90^{\circ}$ a la derecha, $90^{\circ}$ a la izquierda volver a ponerla recta.

Con esta información, el equipo de estudiantes realiza las fases típicas de un Proyecto-Construcción: debe analizar las posibles alternativa de diseño y programación del robot, elegir la solución que creen más adecuada, implementa esa solución, comprobar los resultados obtenidos y exponerlos en clase (oralmente, utilizando el robot en funcionamiento y detallando e algoritmo de programación con el ordenador).

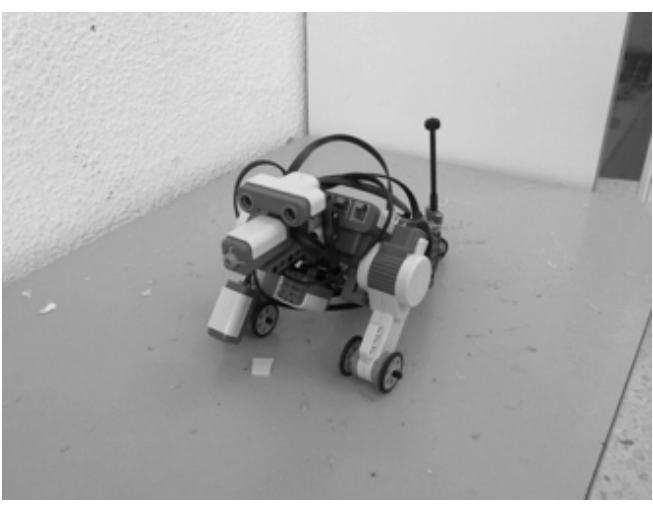

Figura 4. Perro Guardián. Patrulla un pasillo y llama a su dueño cuando una puerta está abierta.

En este proceso, el equipo de trabajo ha tenido que aplicar los conocimientos de robótica adquiridos previamente, investigar críticamente sobre los distintos algoritmos posibles, analizar justificadamente el que consideran mejor e implementarlo con diferentes técnicas: realización de cálculos matemáticos sobre los grados de giro de los motores para conseguir el movimiento de la cabeza del robot, ajuste fino mediante ensayo y error, etc.

Una vez resuelto el primer problema se pasa al siguiente, que tiene un nivel de dificultad mayor. En este caso, una vez que el robot llega al final del primer túnel no solo debe girar la cabeza $90^{\circ}$ a ambos lados sino que debe averiguar en qué lado está el pasillo más corto y hacer que el robot gire hacia el lado correcto. Después se reinicia la marcha y se repite el proceso indefinidamente.

Cuando se resuelve esta situación con un nuevo proceso de Proyecto-Construcción, se plantea el último nivel de dificultad. En este caso, hay que añadir al comportamiento del robot la capacidad de distinguir al final de cada túnel cuando se ha terminado el laberinto por la inexistencia de túneles a ambos lados y hacer que robot finalice su recorrido.

Resolviendo todas las actividades planteadas, los estudiantes aplican de manera completa todas las fases de la metodología Proyecto-Construcción para resolver problemas de dificultad creciente. Además, como el enfoque 
global de la asignatura es la Educación STEM, se sigue un proceso habitual en ingeniería de resolución de problemas y se integran de manera natural Matemáticas, Ciencias y Tecnología: los alumnos están manejando conceptos de Tecnología como relaciones de trasmisión estructuras, etc.; de Física como cálculo de fuerzas o trayectorias; y de Matemáticas como operaciones trigonometricas, graficas y funciones (Ver la figura 5).
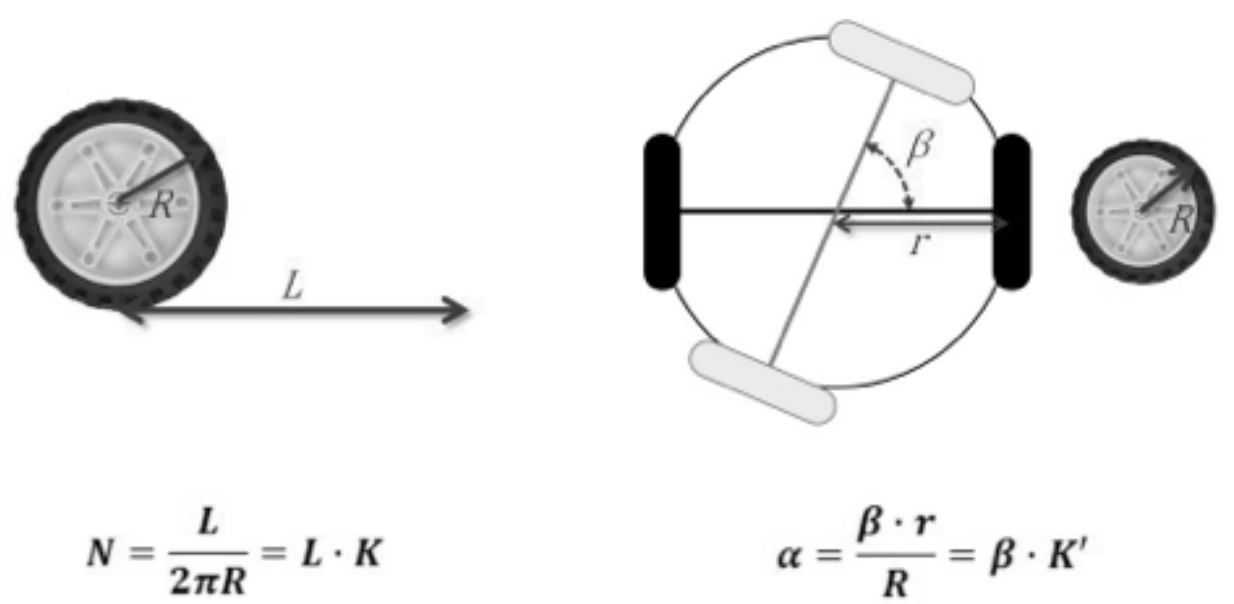

$$
\alpha=\frac{\beta \cdot r}{R}=\beta \cdot K^{\prime}
$$

Figura 5. Relación de magnitudes lineales y circulares para el cálculo de distancias y giros, en función del radio de las ruedas y radio de curvatura.

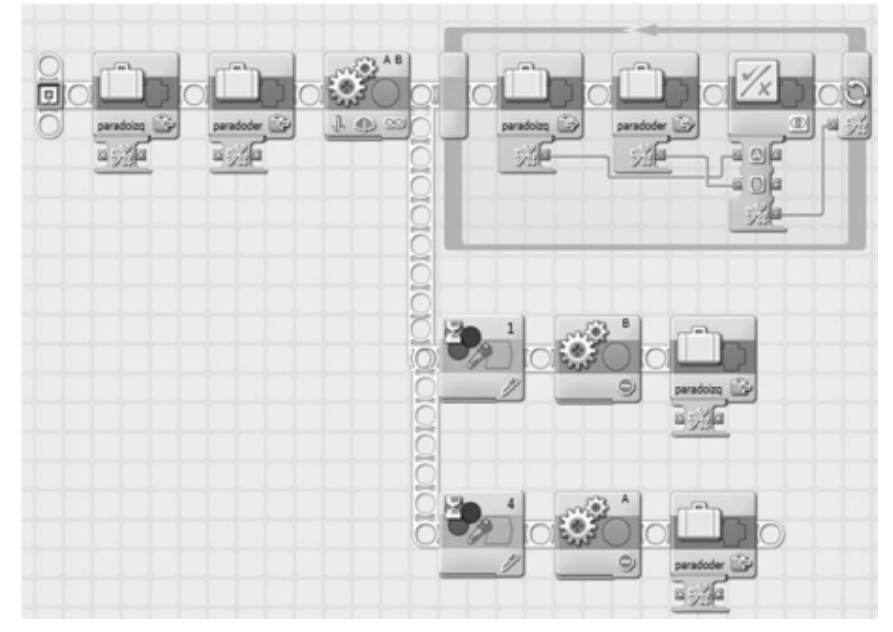

Figura 6. Rutina para colocarse perpendicularmente a una línea negra del suelo: variables, bucle, ejecución en paralelo y operaciones lógicas.

\section{Resultados obtenidos en las}

\section{asignaturas de Robótica}

El éxito de la iniciativa de implantar Robótica como asignatura oficial del currículo en ESO y Bachillerato se puede valorar desde diferentes puntos de vista:

\section{Percepción alumnado}

Desde la implantación de las asignaturas de robótica, la percepción que el alumnado tiene de la Ciencia y la Tecnología ha cambiado notablemente: ven en casa "voluntariamente" documentales de Tecnología, alumnos que en $4^{\circ} \mathrm{d}$ la ESO no tenían intención de estudiar Bachillerato se animan a estudiar incluso el Científico-Tecnológico, todos los que son buenos estudiantes tienen clarísimo que quieren estudia una ingeniería, etc. Un alumno lo resumió diciendo que para ellos es normal este interés por aprender, ya que se habían vuelto unos "frikis de la Tecnología". Lo interesante es resaltar que en clase ahora todos ven "natural" tener interes 作 Tecnología.

\section{Carácter Académico}

La evolución del número de alumnos matriculados en ambas asignaturas es creciente ${ }^{11}$ (Ver figura 7).

También se han mejorado los resultados obtenidos en la Selectividad, estando las notas en Tecnología Industrial de los alumnos del IES Turaniana desde el año 2010 en adelante entre el $9 \%$ de las mejores de la provincia de Almería ${ }^{12}$.

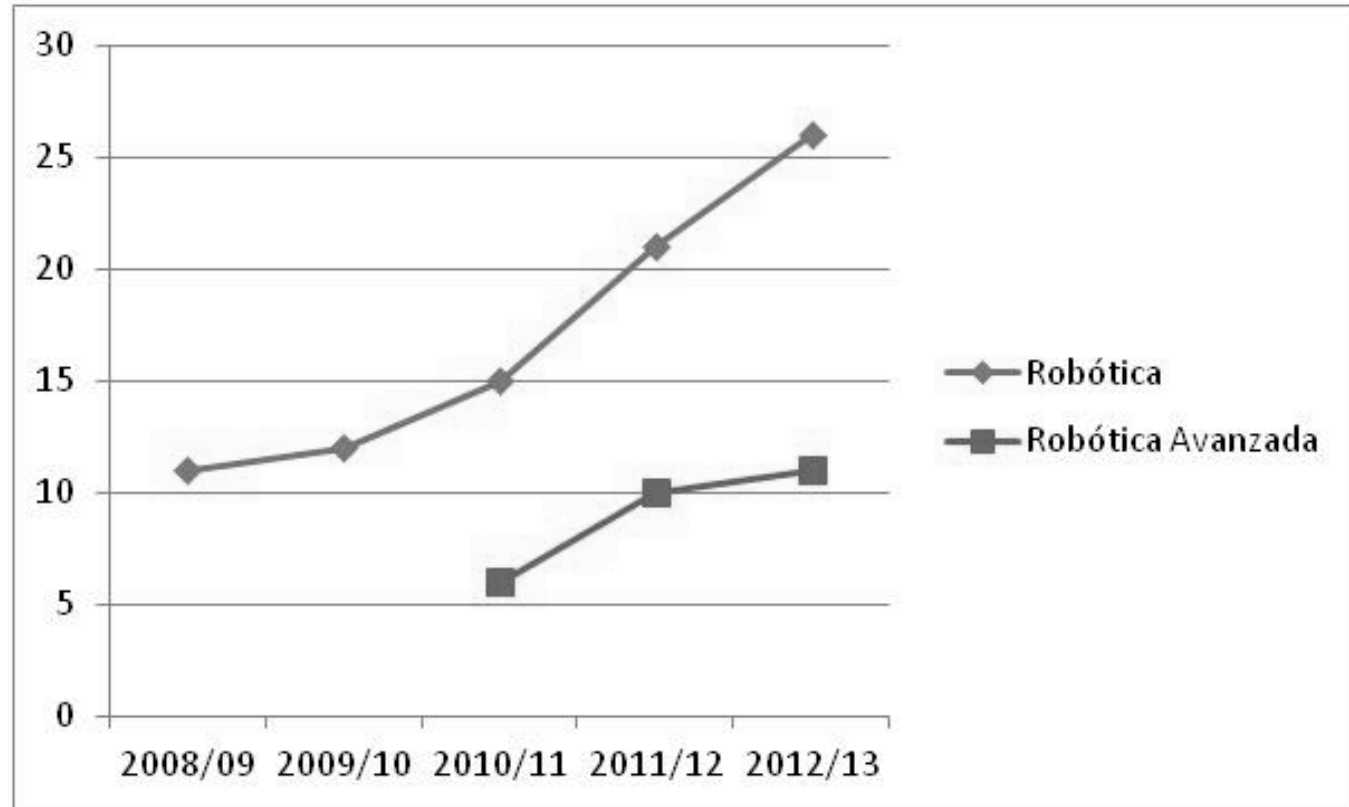

Figura 7. Evolución del nº de alumnos de Robótica.

\footnotetext{
${ }^{11}$ Los datos de matriculaciones se han obtenido de la aplicación SENECA: http://www.juntadeandalucia.es/educacion/ portalseneca/web/seneca/inicio
}

12 Datos obtenidos de las actas de la Ponencia de Tecnología en Almería 
Además, el número de alumnos de Tecnología que al terminar sus estudios de Secundaria continúan sus estudios en Ingeniería en Ciclos Formativos Técnicos de Grado Superior se ha incrementado significativamente desde el año 2009/10, cuando terminó la primera promoción de alumnos de Robótica (Ver la figura 8).

\section{Competición de Robótica FIRST LEGO League}

Desde el punto de vista de valorar los resultados de las asignaturas de Robótica, la FLL sirve como instrumento de evaluación de nuestro planteamiento ya que se trata del único equipo de toda España que plantea el aprendizaje de Robótica como una actividad integrada completamente en el currículo oficial ${ }^{13}$. Los resultados del equipo de competición pueden ser un indicativo de la solidez de la metodología y materiales curriculares propuestos.
Dada la gran cantidad de alumnos y alumnas que están aprendiendo Robótica, nuestra tres equipos: TURABOT Alfa, Beta y Gamma.

La motivación que esta actividad supone es muy llamativa. Dedican voluntariamente y con satisfacción una gran cantidad de horas de su tiempo libre (recreos, guardias, tardes, ...). Un ejemplo de lo que disfrutan preparando la competición es que uno de los días que más les gusta es la "concentración" previa a la competición. Esto supone estar un día completo trabajando, con bocadillos para no salir ni a la hora de comer, y dedicado a hacer los últimos preparativos: ensayos generales, preparación del material de animación (pancartas, banderas, maquillaje, ...), empaquetado de los robots de competición, etc.

También es significativa la autonomía que demuestran a la hora de buscar patrocinadores y conseguir el dinero necesario para la competición: material, inscripción, transporte, alojamiento, etc. En la última edición se consiguieron hasta 35 patrocinadores ${ }^{14}$. participación se ha realizado hasta la fecha con

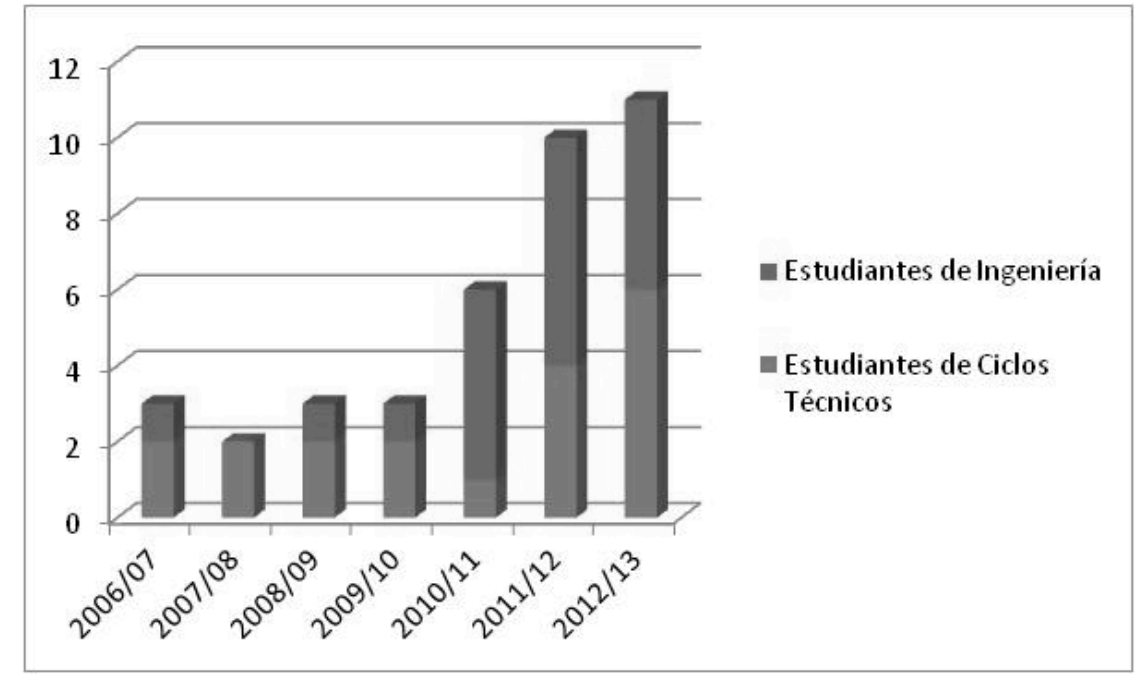

Figura 8. Evolución del nº de estudiantes Post Secundaria.

13 La organización FLL España ha valorado muy positivamente nuestro enfoque y ha hecho un estudio de caso de éxito. También ha encargado la "Guía de Integración Curricular" (Ocaña, 2013), disponible en:

http://www.firstlegoleague.es/docs/FLL/EI_desafio/FSCI_Guia_de_integracion_curricular_130906.pdf

14 Hecho que ha sido destacado a nivel nacional e internacional por la FLL: http://www.firstlegoleague.es/docs/Como_encontrar_financiacion. pdf
Los resultados conseguidos han sido muy satisfactorios, sobre todo teniendo en cuenta que los alumnos que forman los equipos cambia todos los años: Tricampeones FLL Almería, tre veces clasificados para el Campeonato de España consiguiendo un $5^{\circ}$ puesto y ser Subcampeone de España en 2013, ganadores de 11 Trofeos (dos a nivel nacional) y clasificados para el Campeonato del Mundo en los cursos 2011/12 y 2013/14.

Una prueba de la importancia social de estos resultados es el gran impacto mediático que el equipo Turabot ha tenido en prensa, radio y televisión (Ocaña, 2010, 2011).

\section{Conclusiones}

Podemos expresar nuestra valoración positiva sobre la creación y puesta en marcha de las nuevas asignaturas de Robótica.

Los alumnos que cursan estas asignaturas se muestran en general motivados y satisfechos, in distintamente del recorrido de aprendizaje que hayan seguido. Una prueba de ello es que todo los alumnos que cursan la asignatura en $4^{\circ}$ de la ESO y continúan sus estudios en el Bachillerato Científico-Tecnológico, se matriculan de nuevo en la optativa "Robótica Avanzada".

También es destacable el desarrollo conseguido en habilidades y capacidades que no so propias de ninguna asignatura del currículo y que resultan difíciles de conseguir: resolución de problemas, creatividad, autonomía e iniciativa personal, trabajo en equipo, motivación aprendizaje colaborativo, etc.

Y también consideramos muy significativos los resultados obtenidos en la competició FIRST LEGO League. Conseguir de manera continuada trofeos tanto a nivel provincial como nacional, y obtener dos clasificaciones para la fase mundial teniendo en cuenta que todos los años cambian los componentes de equipo, es un hecho que nos sirve como una valoración externa y objetiva de las competencia que pretendemos y del proceso de enseñanzaaprendizaje que proponemos.

Para terminar, proponemos varias líneas de trabajo futuras.
Como la Robótica es una disciplina intrínsecamente multidisciplinar, se puede profundizar aún más en la integración de la Robótica Educativa con otros bloques de conocimientos del currículo oficial de Educación Secundaria: electrónica analógica y digital, energías renovables, hidráulica, neumática, informática, etc.

Por otro lado, tras comprobar que el material también resulta atractivo y motivador para alumnado que no es de Ciencias y Tecnología, podría plantearse la asignatura de Robótica en todos los cursos de la ESO e incluso en los últimos cursos de Primaria $\left(5^{\circ}\right.$ y $6^{\circ}$, ya que los robots LEGO Mindstorms son para niños a partir de diez años).

También se ha comentado el desarrollo que adquiere el alumnado de algunas habilidades de carácter transversal. Sería interesante investigar y dar evidencias de que, efectivamente, la Robótica Educativa contribuye de manera significativa al desarrollo de estas habilidades.

Por último, tras comprobar la facilidad para que cada tipo de alumno desarrolle sus habilidades a su propio ritmo, también se propone indagar en las posibilidades de la Robótica Educativa como herramienta de atención a la diversidad. De manera que la creación de actividades diseñadas específicamente con este fin permita desarrollar al máximo las capacidades, tanto de alumnado con necesidades de medidas de refuerzo educativo como los diagnosticados de altas capacidades.

\section{REFERENCIAS}

Alimisis, D. y Kynigos, C. (2009). Constructionism and Robotics in Education. En Alimisis, D. (ed.), Teacher education on robotics-enhanced constructivist pedagogical methods (pp. 11-26). Atenas: ASPETE

Bruning, R. H., Schraw, J. G., Norby, M. M., y Ronning, R. R. (2004). Cognitive psychology and instruction. Columbus: Pearson.

Bransford, J. D., Brown, A. L., y Cocking, R. R. (2000). How people learn: Brain, mind, experience, and school. Washington: National Academy Press. 
Capraro, R. M., Capraro, M. M., Morgan, J. y Scheurich (2010). A companion to interdisciplinary STEM project based learning: for teach ers by teachers. Rotterdam: Sense Publishers.

Capraro, R. M. y Slough, S.W. (2009). Projectbased learning. An integrated science, technology, engineering, and mathematics (STEM) ap proach. Rotterdam: Sense Publishers.

Cortés, J. A., Arbeláez, O., y Mendoza, J. A. (2009) El entorno LEGO Mindstorms en la introducción a la robótica y la programación. Scientia et Technica, 41(1), 42-45.

Criado, A. M. y García, A. (2011). Las experiencias prácticas para el conocimiento del medio natural y tecnológico en la formación inicial de maestros. Investigación en la Escuela, 74 73-88.

Consejería de Educación (2007). Decreto 231/2007, de 31 de julio, por el que se establece la ordenación y las enseñanzas correspondientes a la educación secundaria obligatoria en Andalucía. Sevilla: Boletín Oficial de la Junt de Andalucía.

Consejería de Educación (2008). Decreto 416/2008, de 22 de julio, por el que se establece la ordenación y las enseñanzas correspondientes al Bachillerato en Andalucía. Sevilla: Boletín Oficial de la Junta de Andalucía.

Drew, D. (2011). STEM the tide. Reforming science, technology, engineering and math education in America. Baltimore: The Johns Hopkins University Press.

Fisher, P., Zeligman, D. y Fairweather, J. (2005) Self-assessed student learning outcomes in an engineering service course. International Journal of Engineering Education, 21, 446-456.

Fortus, D., Krajcikb, J., Dershimerb, R. C., Marx R. W. y Mamlok-Naamand, R. (2005). Design-based science and real-world problem solving. International Journal of Science Education, 27(7), 855-879.

Grant, M. M. (2002). Getting a grip on projectbased learning: theory, cases and recommendations. Meridian: A Middle School Compute Technologies Journal, 5(1). Recuperado septiembre 1, 2012 de http://www.ncsu.edu/meridian/win2002/514/3.htm

Gura, M. (2011). Getting started with LEGO robot ics. A guide for K-12 educators. Oregon: International Society for Technology in Education.
Harland, D. J. (2011). STEM. Student research handbook. Arlington: National Science Teachers Association Press.

Hartzler, D. S. (2000). A meta-analysis of studies conducted on integrated curriculum programs and their effects on student achievement. Tesis Doctoral: Indiana University

Hernández, F. y Ventura, M., (1992). La Organización del currículum por proyectos de trabajo. Barcelona: Grao.

Kelly, J. y Daudelin, J. (2008). First LEGO League: The unofficial guide. San Francisco: No Starch Press.

Kolodner, J. L., Camp, P. J., Crismond, D., Fasse, B., Gray, J., Holbrook, J., et al. (2003). Problembased learning meets case-based reasoning in the middle-school science classroom: putting learning by design into practice. The Journal of the Learning Sciences, 12(4), 495-547.

Laboy-Rush, D. (2011). Integrated STEM education through project-based learning". Portland: Learning.com. Recuperado enero 23, 2012 de http://www.learning.com/stem/whitepaper

Lacueva, A. (2008). Integrar para educar mejor: posibilidades y exigencias. Investigación en la Escuela, 66, 43-53.

Mastascusa, E., Snyder, W.J. y Hoyt, B.S. (2011). Effective instruction for STEM disciplines. From learning theory to college teaching. San Francisco: Jossey-Bass.

Ministerio de Educación (2007a). Real Decreto 1631/2006, de 29 de diciembre, por el que se establecen las enseñanzas mínimas correspondientes a la Educación Secundaria Obligatoria. Madrid: Boletín Oficial del Estado.

Ministerio de Educación (2007b). Real Decreto 1467/2007, de 2 de noviembre, por el que se establece la estructura del bachillerato y se fijan sus enseñanzas minimas. Madrid: Boletín Oficial del Estado.

Ocaña, G. (2010). Robótica Educativa. Recuperado Abril 7, 2013 de http://www.youtube.com/ user/gocanar

Ocaña, G. (2011). Robótica Educativa. Perfil en redes sociales. Recuperado enero 25, 2013 de http://www.facebook.com/robotica.turaniana y http://twitter.com/Turabot

Ocaña, G. (2012). Robótica en el Aula. Almería: Consejería de Educación de la Junta de Andalucía.
Ocaña, G. (2013). Guía de Integración Curricular. Fundación Scientia. FIRST LEGO League. Recuperado Marzo 5, 2013 de http://www.firstlegoleague.es/wp-content/uploads/2015/09/ DocTT/FLL_TT15_Guia_integracion_curricular_151005.pdf

Papert, S. (1980). Mindstorms: children, com puters, and powerful Ideas. New York: Basic Books.

Pisciotta, M., Vello, B.; Bordo, C., y Morgavi, G. (2010). Robotic competition: A classroom experience in a vocational school. En A. Kallel, A. Hassairi, C.A. Bulucea y N. Mastorakis (Eds.) Actas del 6th International Conference on Educational Technologies (pp. 151-156). Kantaoui, Tunez: WSEAS Press

Pozuelos, F. J. y Rodríguez F. P. (2008). Trabajando por proyectos en el aula. Aportaciones de una investigación colaborativa. Investigación en 1 Escuela, 66, 5-27.

Ruiz-Velasco, E. (2007). Educatrónica. Innovación en el aprendizaje de las ciencias y la tecnología. Madrid: Díaz de Santos.
Sanders, M. (2009). STEM, STEM Education, STEMmania. The Technology Teacher, 69(4), 20-26.

Satchwell, R. y Loepp, F. L. (2002). Designing and implementing an integrated mathematics, science, and technology curriculum for the middle school. Journal of Industrial Teacher Education, 39(3). Recuperado julio 17, 2012 de http://scholar.lib.vt.edu/ejournals/JITE/ v39n3/satchwell.html

Schön, D. A. (1987). Educating the reflective practitioner: toward a new design for teaching and learning in the professions. San Francisco: Jossey-Bass.

Thomas, J. W. (2000). A review of research on project-based learning. San Rafael: Buck Institute for Education.

Wai, J., Lubinski, D., Benbow, C. P., y Steiger J. H. (2010). Accomplishment in science, technology, engineering, and mathematics (STEM) and its relation to STEM educational dose: A 25-year longitudinal study. Journal of Educational Psychology, 102(4), 860-871. 